Editorial

\title{
Preeclampsia: New Concepts and Approach of a National Protocol
}

\author{
Ricardo de Carvalho Cavalli ${ }^{10}$ Henri Augusto Korkes² \\ 1 Departament of Gynecology and Obstetrics, Faculdade de Medicina, \\ Universidade de São Paulo, Ribeirão Preto, SP, Brasil \\ ${ }^{2}$ Departament of Obstetrics, Pontifícia Universidade Católica de São \\ Paulo, São Paulo, SP, Brasil
}

Rev Bras Ginecol Obstet 2019;41:277-279.

\section{Importance of the Topic and Development of a National Protocol}

Hypertensive syndromes, along with hemorrhagic syndromes, account for a large share of maternal deaths worldwide. In developing countries such as Brazil, maternal deaths are mostly related to hypertensive complications, and preeclampsia (PE) plays a prominent role in this scenario. ${ }^{1,2}$ Several factors contribute to these outcomes, such as late diagnosis, low rates of prescription of pharmacological preventive methods, inadequate prenatal care both in number and quality of visits, low and inadequate use of magnesium sulfate in the prevention of eclampsia, and poor postpartum follow-up. These factors, individually or together, can exert negative influence and increase maternal morbidity and mortality rates in our country.

The difficulty in understanding PE in terms of its etiology, pathogenesis and clinical presentation is already known. However, this aspect is aggravated because each country, region and even city services denominate and classify hypertensive syndromes in ways that generate confusion among colleagues, which delays the diagnoses and conducts. With the purpose of combating these known and undesirable difficulties, unifying the care provided to pregnant women, and reducing maternal death rates in our country, it was essential to create a national protocol on preeclampsia.

\section{Development of the National Protocol}

Given the great importance of hypertensive syndromes in maternal and fetal morbimortality in Brazil, in 2016, the Brazilian Federation of Gynecology and Obstetrics Associations (Federação Brasileira das Associações de Ginecologia e Obstetrícia, Febrasgo, in the Portuguese acronym) reactivated the National Specialized Commission on Hypertension

(1) Ricardo de Carvalho Cavalli's ORCID is https://orcid.org/00000001-5010-4914.

Address for correspondence Ricardo Carvalho Cavalli, Av. Bandeirantes 3900, 14049-900, Vila Monte Alegre, Ribeirão Preto, SP, Brazil (e-mail: rcavalli@fmrp. usp.br). in Pregnancy (CNE-Hypertension; Comissão Nacional Especializada [CNE] Hipertensão na Gestação, in Portuguese). Currently, it is composed of 15 members from different universities dispersed throughout Brazil who are specialized in the topic. The process of developing the national protocol began strongly in 2016 and has proved to be a real challenge from the start, because, although knowledge on PE has advanced over the last decade, many questions remain. We were also faced with different protocols in the different medical-hospital services that reflect a recent transition in knowledge and understanding about the subject. Different procedures regarding nomenclature, medications used, requested tests and the timing of solution of PE cases were just some of the points addressed throughout the development process. The initial CNE-Hypertension meetings were held electronically and remotely, but face-to-face discussions were necessary for some definitions. Hence, in-person meetings were scheduled by taking advantage of scientific events in our country, in which most members would already be present, such as national, regional and international congresses. The most recent guidelines published so far and the scientific works of greatest impact in this area were considered for the development of this protocol. Key protocols were reviewed, namely those of the American College of Obstetricians and Gynecologists (ACOG), the International Society for the Study of Hypertension in Pregnancy (ISSHP), the World Health Organization (WHO), the Royal College of Obstetricians and Gynecologists (RCOG), the International Federation of Gynecology and Obstetrics (FIGO) and the United Kingdom's National Institute for Health and Care Excellence (NICE). Thus, the Protocol for the Management of Preeclampsia developed by Febrasgo through the CNEHypertension began. In this constructive process, various specialists of each service in Brazil were able to look at the current world knowledge about the pathology and adapt it to the Brazilian reality in a homogeneous way. At the same time that novelties and new technologies emerged, it was necessary to look at our reality and think about their applicability

Copyright (e 2019 by Thieme Revinter Publicações Ltda, Rio de Janeiro, Brazil
DOI https://doi.org/ 10.1055/s-0039-1688959. ISSN 0100-7203.
License terms

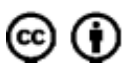


in our environment by trying to aggregate scientific knowledge with feasibility. This issue will contemplate the Febrasgo statement.

\section{Relevant Aspects of the Diagnosis}

We are fully convinced that an early diagnosis can lead to lower maternal mortality from PE in our country. In practice, many cases go undetected and are only diagnosed after more severe complications. Perhaps because of lack of resources or no suspicion of the condition, patients are often released home with PE already installed, but unidentified by the assistant team. A careful prenatal care with attention to the patient's complaints, weight gain, edema and correct evaluation of pressure levels, is a very valuable weapon in this war against maternal mortality from PE. An important element is the correct measurement of blood pressure (BP) levels that considers the correct position of the pregnant woman, and the adequate cuffs for each patient. If there is clinical suspicion of PE, it is essential to rule out the diagnosis; hence, patients should undergo specific tests. Some patients with PE and possibly severe complications will not present proteinuria; therefore, in the current recommendation in our protocol, the presence of proteinuria was not obligatory for suspected cases with clinical and/or laboratory deterioration. In our protocol, the choice was to bring to our milieu the concepts presented by the ACOG initially in $2013,{ }^{3}$ and again in 2019, ${ }^{4}$ and some concepts from the ISSHP published in $2018,{ }^{5}$ regarding the diagnostic criteria for PE. To avoid losing time until the diagnosis and by considering the complex pathophysiology of preeclampsia in our country, we advise establishing the diagnosis even in the absence of proteinuria, when the patient presents BP elevation associated with specific alterations in target organs, ${ }^{4}$ or signs of maternal ${ }^{4}$ or fetal deterioration. ${ }^{5}$

\section{Prediction - Prevention}

Considering the occurrence of PE in the general population ( 2 to $5 \%$ ), most predictive methods do not offer reasonable sensitivity. As prediction tests are not yet part of the reality of care in our country, the differentiated prenatal care with directed anamnesis and search of risk groups for PE remains the most effective and feasible method to provide preventive care for the patients. This aspect was greatly discussed in our committee, given the recent publications on combined methods for predicting PE performed in the first quarter of 2019. As no method or combination of methods can detect all cases of $\mathrm{PE}$, and because of the high cost and low clinical experience, currently, according to recommendations of the $\mathrm{WHO}^{6}$ and $\mathrm{ACOG},{ }^{4}$ we chose to keep our guidelines based solely on the patients' medical history. In cases of patients with risk factors for PE, we recommend the use of acetylsalicylic acid (ASA) and calcium as early as possible (preferably before 16 weeks) until the end of pregnancy (at least up to 36 weeks). The best available evidence currently points to the use of low doses of ASA (100 to $150 \mathrm{mg} /$ day $)^{6}$ and the use of calcium
(1.0 to $2.0 \mathrm{~g} / \mathrm{day})^{7}$ as genuinely beneficial interventions in groups at risk of developing PE.

\section{Patient Follow-up after Diagnosis}

The management of patients with preeclampsia, including the best time for delivery, was one of the most controversial subjects discussed in our committee. According to the current understanding of the disease, the guidance is that PE cases with clinical and/or laboratory deterioration should be referred for resolution due to high maternal risk. In contrast, controlled cases from the maternal and fetal point of view without worsening in clinical and laboratory parameters should normally be monitored for a longer time. Obviously, this aspect of the expectant management of patients with PE is mainly due to inherent risks of prematurity. Cases of PE close to term, especially with signs of severity, indicate that the pregnancy should be interrupted. After the diagnosis of PE in cases that will be monitored, a plan of action directed to maternal and fetal surveillance should be implemented and include laboratory tests and a periodic assessment of fetal vitality. Given the high rates of cesarean sections in Brazil, in this protocol, special attention is drawn to the choice of birth route, which should have obstetric indication. Another important contribution in our protocol was the introduction of the "risk calculator", or the preeclampsia integrated and estimated risk (PIERS) mathematical model into the clinical practice. This very important tool can be an ally for decision-making in PE by aiming at reducing the morbidity and mortality of our patients. ${ }^{8}$ In this mathematical model, the clinical and laboratory parameters to calculate the risk of adverse outcomes within 48 hours from the time of admission of the patient with PE are considered. The inclusion of a tool that can guide our decisions more objectively appears to be critical in terms of maternal and fetal protection. Although there is no specific value of PIERS results indicating intervention, the decision will be according to the care scenario and by bearing in mind that a patient at $1.5 \%$ risk is totally different from a calculated risk of $26.5 \%$, for example.

\section{Pharmacological Aspects}

In this topic, the protocol presents specific updates of medications available in our country to treat hypertension in pregnancy, as well as in hypertensive crises and the postpartum period by bringing tables with the administration of each drug in a simple and practical way. The document also emphasizes the importance of a more liberal use of magnesium sulfate in severe cases, such as: imminent eclampsia, eclampsia, hemolysis, elevated liver enzymes, and low platelet count (HELLP) syndrome, PE with signs of severity, or clinical and/or laboratory deterioration.

At this stage, the Febrasgo's CNE-Hypertension protocol is already a reality. All efforts are being made so that this knowledge - which is extremely aligned with international practices, based on the best scientific evidence to date, and adapted to the Brazilian reality - reaches all professionals who assist pregnant patients. In order to make this possible, the Brazilian Ministry of Health must adopt this document as 
a national protocol, so that together we can lower the rates of maternal death in our country.

Conflicts of Interest

The authors have none to disclose.

\section{References}

1 von Dadelszen P, Payne B, Li J, et al; PIERS Study Group. Prediction of adverse maternal outcomes in pre-eclampsia: development and validation of the fullPIERS model. Lancet 2011;377 (9761):219-227. Doi: 10.1016/S0140-6736(10)61351-7

2 Duley L. The global impact of pre-eclampsia and eclampsia. Semin Perinatol 2009;33(03):130-137. Doi: 10.1053/j.semperi. 2009.02.010

3 American College of Obstetricians and Gynecologists. Task Force on Hypertension in Pregnancy. Hypertension in Pregnancy. Washington, DC: ACOG; 2013https://www.acog.org/ /media/Task\%20Force \%20and\%20Work\%20Group\%20Reports/public/HypertensioninPregnancy.pdf. Accessed: March 20, 2018.
4 ACOG Practice Bulletin No. 202 Summary: gestational hypertension and preeclampsia. Obstet Gynecol 2019;133(01):211-214. Doi: 10.1097/AOG.0000000000003019

5 Brown MA, Magee LA, Kenny LC, et al; International Society for the Study of Hypertension in Pregnancy (ISSHP). Hypertensive disorders of pregnancy: ISSHP classification, diagnosis, and management recommendations for international practice. Hypertension 2018; 72(01):24-43. Doi: 10.1161/HYPERTENSIONAHA.117.10803

6 World Health Organization. WHO Recommendations for Prevention and Treatment of Pre-Eclampsia and Eclampsia. 2011http:// apps.who.int/iris/bitstream/10665/44703/1/9789241548335_eng. pdf. Accessed: June 13, 2018.

7 Hofmeyr GJ, Lawrie TA, Atallah AN, Duley L, Torloni MR. Calcium supplementation during pregnancy for preventing hypertensive disorders and related problems. Cochrane Database Syst Rev 2014;(06):CD001059. Doi: 10.1002/14651858.CD001059.pub4

8 von Dadelszen P. M11.1 Identifying risk in women with preeclampsia: the validated PIERS model. Pregnancy Hypertens 2010;1:S8. Doi: 10.1016/S2210-7789(10)60045-1 